in vivo $34: 3731-3734(2020)$

doi:10.21873/invivo.12222

\title{
Radiotherapy in the COVID-19 Pandemic Era
}

\author{
ALESSANDRA GONNELLI $^{1}$, SABRINA MONTRONE ${ }^{1}$, PAOLA COCUZZA ${ }^{2}$, STEFANO URSINO ${ }^{1}$, \\ BRUNO MANFREDI ${ }^{1}$, ROBERTO MATTIONI ${ }^{1}$, NATALINA COCCIA ${ }^{1}$, TAIUSHA FUENTES ${ }^{1}$, \\ ELISA CALISTRI ${ }^{1}$, FRANSCESCO PASQUALETTI $^{1}$ and FABIOLA PAIAR ${ }^{1}$ \\ ${ }^{1}$ Department of Radiation Oncology, Azienda Ospedaliero Universitaria Pisana, Pisa, Italy; \\ ${ }^{2}$ Department of Radiotherapy, San Luca Hospital, Lucca, Italy
}

\begin{abstract}
Background/Aim: In 2020, because of coronavirus pandemic, medical activities changed. The aim of this report is to compare the volumes of Pisa radiotherapy activities from March $9^{\text {th }}$ to May 31 ${ }^{\text {st }}, 2020$, with the same period in 2019. Patients and Methods: We analyzed the activity of our Unit to evaluate how logistics changes, related to the COVID-19 epidemic, impacted on volumes of radiotherapy $(R T)$ activity and on the number of cases of COVID-19 infections observed in healthcare professionals and patients. Results: The total number of first-time visits between March-May 2020 was reduced by $18 \%$, probably due to delays in diagnosis and histological tests as well as the temporary closure of the operating rooms. None of the healthcare professionals and only two patients contracted the infection. Conclusion: We were able to treat all patients referred to our hospital and we were able to reduce risk of infection for both our patients and healthcare staff, guaranteeing continuum of care for our oncological patients.
\end{abstract}

In December 2019 the World Health Organization (WHO) China Country Office informed of cases of pneumonia of unknown etiology detected in Wuhan city (1). It was a starting point of a worldwide COVID-19 pandemic caused by an RNA virus belonging to the family of Coronaviridae. The first Italian COVID-19 patient (pt) in a hospital ward, which triggered the national emergency, was diagnosed in Codogno, in the south of Milan, on the $20^{\text {th }}$ of February in 2020. Initially, the Italian government defined three geographical areas with a different risk of infection; however, on March 9, 2020, due to the spread of the infection, it was decided to extend the measure of the high-

This article is freely accessible online.

Correspondence to: Alessandra Gonnelli, Department of Radiation Oncology, Azienda Ospedaliero Universitaria Pisana, Via Roma 67, 56100 Pisa, Italy. Tel: +39 3404844179, e-mail: gonnelli. alessandra@gmail.com

Key Words: COVID-19, pandemic, radiotherapy, cancer. risk zones to the entire national territory, placing the country under complete lockdown.

All public hospitals tackled the emergency with radical changes in wards' organization. Almost all Italian hospitals shifted the health care workers and activities on the treatment of COVID-affected pts, preserving only life-saving activities, such as cancer treatments, such as radiotherapy (RT), which plays a pivot role in tumor management. Indeed, RT represents an important step in the cure of oncology pts, as $60-70 \%$ of all cancer pts are treated with RT (from curative to palliative intent) (3). It is considered a "life-saving" activity and should be guaranteed to all pts for whom it is indicated. Moreover, it is "time-sensitive" and RT interruption is associated with poor local control (4). Ensuring the continuum of cancer care is a cornerstone for oncology, and this has become a major priority during the COVID-19 pandemic. Therefore, RT departments have continued to regularly perform the most of activities during the emergency (5).

Our service, the Operative Unit of Radiotherapy (OUR) of the University Hospital of Pisa, is characterized by a large and heterogeneous cohort of pts. During the past year (January 2019-December 2019) we have examined 16,000 pts (first-time clinical evaluation or regular follow-up visit) of who we treated $1,800 \mathrm{pts}$, and hospitalized 175 pts. Moreover, 790 pts of these were evaluated during the hospitalization for the opportunity of an RT treatment.

During the COVID-19 pandemic we had to continue providing our services while protecting pts, families of the pts and our staff (12 radiation oncologists, 9 interns, 6 medical physicists, 13 radiation therapists, 9 nurses and 3 hospital administrative staff) from COVID-19 infection. Our aim here was to describe our activities and underline the solutions and pitfalls of the RT management during the COVID-19 pandemic era.

\section{Patients and Methods}

We retrospectively analyzed the activity of our OUR at the University Hospital of Pisa from March $9^{\text {th }}$ to May 31 $1^{\text {st }}, 2020$ (and compared it to the activity of the same period in 2019 with direct statistical comparison) to evaluate how logistics changes, related to 
the COVID-19 epidemic, impacted on the volume of RT activity and on the risk of infection for patients and health professionals

Our team, following hospital directives, assembled measures that allowed us to better face the emergency. For both pts and health professionals that it was mandatory to wear surgical masks inside the hospital, according to the indications continuously updated by the WHO.

The staff of OUR was limited but without interrupting our services; people in positions of hospital administration switched into smart working mode and interns were kept out of the RT unit for two weeks in rotation.

All staff were tested using serology testing for COVID-19, whereas a nasopharyngeal swab was performed in case of suspected COVID-19 symptoms or if in close contact with a confirmed COVID-19 pt (6).

To reduce the risk of hospital contagion we made changes in our daily routine in order to guarantee pts an optimal service. In particular, follow-up visits were postponed if not regarded essential following counseling by phone with a radiation oncologist.

The day before the access to the UOR, for patients with programmed first access Computer Tomography (CT) simulation and for the beginning of treatment, nurses performed telephone screening with a questionnaire checking the presence of any respiratory symptoms or social contacts with COVID-19-positive or suspected pts.

Once pts arrived at our department, a triage point at the entrance carried out a second screening using a temperature check. Patients with planned hospitalization or day-hospital access were tested using a nasopharyngeal swab to confirm the therapeutic pathway. Waiting rooms were reorganized in order to maintain social distancing (at least 1 meter) with signs placed on chairs to indicate where to sit and where not to. Hand sanitizer gel was available in both waiting and treatment rooms. When possible, entry of accompanying persons was forbidden. Hypofractionation regimen was considered in order to reduce fractions of radiotherapy and, consequently, the number of pt access, as it is given over a shorter period of time compared to standard radiation therapy.

Between each pt the linear accelerator (LINAC) and RT equipment were sanitized. Radiotherapy for pts with a better prognosis, such as adjuvant radiation therapy for pts with breast and prostate cancer, radical radiation therapy for pts with low risk prostate disease as well as radiation for benign and functional diseases was postponed.

Finally, interdisciplinary case tumor boards were organized via video conferences to avoid gathering of people.

\section{Results}

We tracked down volumes of our activities from March $9^{\text {th }}$ to May $31^{\text {st }}, 2020$, and compared them to volumes during the same period in 2019 .

Compared to 2019 , we observed an $18 \%$ reduction in first access pts (480 pts in 2019 and 390 pts in 2020) and a 5\% increase (346 treatments in 2019 and 365 in 2020) in RT treatments.

As shown in Figure 1, we found an increase in pts evaluated for palliative treatment and otorhinolaryngologic (ORL) tumors and a reduction for the rest.

COVID-19 infection in oncology healthcare professionals. Among a total of 52 healthcare professionals (12 radiation oncologists, 9 interns, 6 medical physicists, 13 radiation therapists, 9 nurses and 3 hospital administration staff) we reported no cases of COVID-19 infection.

COVID-19 infection in active oncological pts. Among the 365 pts treated, only two pts contracted the infection. The first pt (CM) was having a stereotactic treatment for a nodal recurrence from prostate cancer, while the second one (FC) came for a conventional treatment for lung cancer (30 sessions of RT in combination with chemotherapy CT).

$\mathrm{CM}$ was asymptomatic and was tested using a swab on account of a previous contact with an infected pt.

FC presented fever, followed by cough, which developed during treatment. The pt was not hospitalized and managed the illness at home.

For the first pt, the COVID-19 positivity was communicated when the treatment was already completed. Instead, for the second pt, the positivity was communicated during RT, which led to a cumulative dose of $27 \mathrm{~Gy}$ and a short interruption of treatment. RT was resumed 25 days later, when two consecutive tests resulted as negative.

\section{Discussion}

Careful organisational measures allowed for a minimal reduction in the volumes of RT activities. Implementation of initial telephone consultation and presence of triage, additional remote consultation activities, reduction of hospital access and social distancing policies were widely accepted by pts and minimally interfered with the effective delivery of cancer treatments. The absence of infections in the healthcare staff and the low incidence of infection $(0.54 \%)$ between pts suggest that the organisational and protective measures, in agreement with the institutional directives, were fundamental to minimize the spread of infection. However, we realized that some types of personal protective equipment (PPE) were not sufficient considering the heterogeneity of the pts. For example, for pts with head-neck and lung cancer, FFP2 masks, eye protection, such as goggles or disposable face shields covering the front as well as the sides of the face would be more useful. Unfortunately, only surgical masks and disposable gloves were provided to healthcare professionals. Moreover, in addition to the healthcare staff, it would be useful to test all the pts for COVID-19 infection.

In the analyzed trimester of 2020,390 pts acceded to our service for a first clinical evaluation and 365 pts underwent RT.

From an analysis of subgroups of pts who accessed OUR we identified a reduction in first-time RT visits for some pathologies compared to last year.

We hypothesized that the overall reduction in first-time access could be due to diagnostic delays (endoscopic and radiological examinations) as well as delays in the 


\section{Comparison of activities 2019-2020}

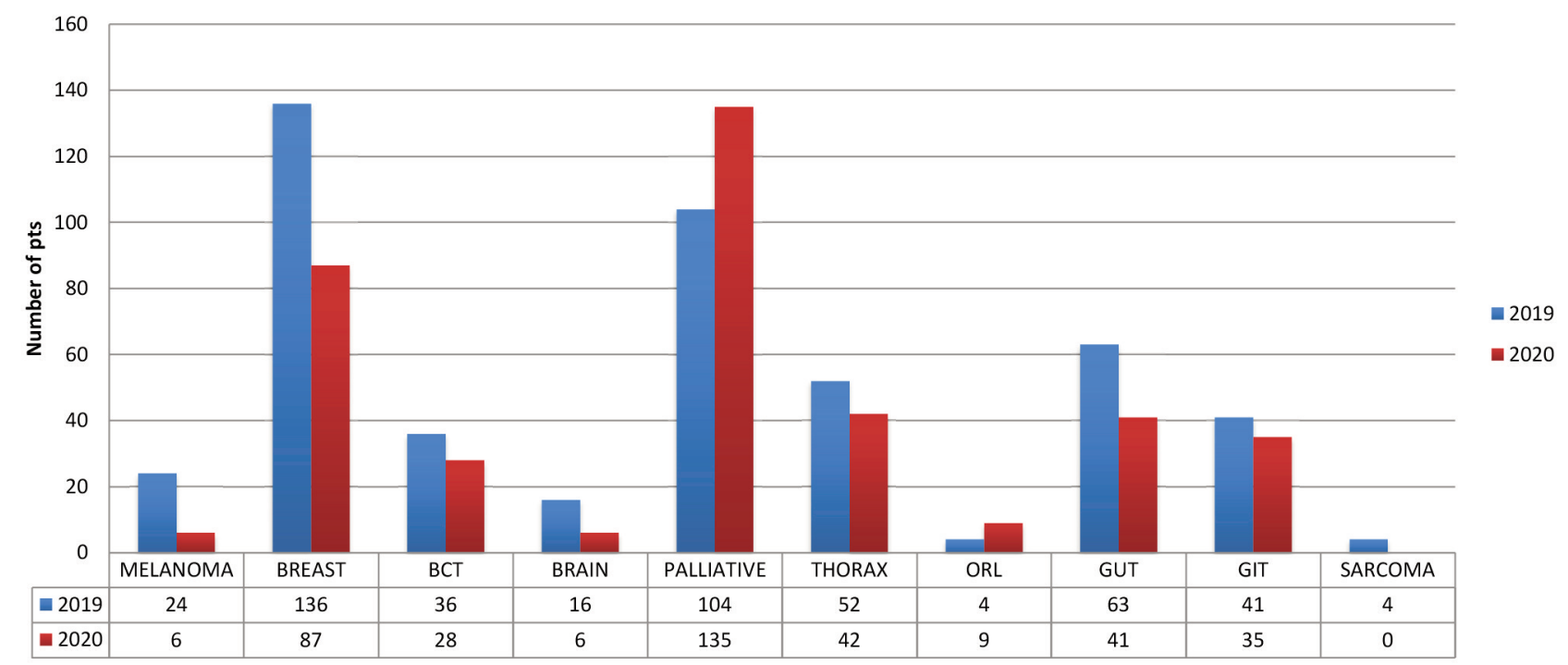

Figure 1. Comparison of activities 2019-2020.

completion of histological/cytological tests due to staff reduction caused by COVID- 19 .

The temporary closure of the operating rooms also contributed to the reduction of access for patients who could need postoperative treatment.

For patients with breast or prostate cancer (in the case of postoperative treatment or low-risk cancer), RT was identified as a deferrable treatment according to national guidelines (5), therefore, the access of patients with these pathologies was reduced.

The reduction of pts with thoracic and gastrointestinal malignancies correlated with a delay in diagnosis due to reductions in screening and radiological or endoscopic exams. Patients with brain cancer also presented with lower numbers compared to 2019. This can be related to a reduction in the number of planned surgeries, considering that the majority of our central nervous system (CNS) cancer pts underwent surgery in Milan (Lombardy is the Italian region more involved in this pandemic).

Moreover, the reduced numbers of pts with uveal melanoma and sarcoma can be correlated to the reduction in the number of patients from other Italian Regions, considering that the Azienda Ospedaliero Universitaria Pisana (AOUP) is one of the national reference centers for these pathologies.

Instead, we noticed that pts with head and neck cancer slightly increased compared to 2019. Usually pts with an earlystage head and neck cancer (e.g. glottic cancer) are treated with one modality, either RT or surgery, both of which can offer a similar control of the disease. During this period, due to the reduction in the availability of surgery rooms, RT was preferred.
The other category in which we observed an increase was visits for palliative treatment. These are usually hypofractioned, which could explain the increase in the number of treatments at the LINAC.

The increase of palliative treatments happened probably due to a reorganization between COVID and non-COVID19 departments that resulted in the temporary closure of some activities, such as in palliative therapy and surgery departments (the anesthesiologists were mostly recruited to assist COVID patients in intensive therapy units).

In conclusion, in spite of the difficulties during the pandemic era, we were able to treat all the pts referred to our hospital, following national and international guidelines. Although we faced a new unknown health emergency, we were satisfied with the management and organization in our department. Indeed following the ministerial directives we have been able to guarantee health professionals safely, without infections; at the same time we were able to guarantee the continuity of the oncological treatment.

\section{Conflicts of Interest}

No potential conflict of interest was reported by the Authors.

\section{Authors' Contributions}

FP conceptualized the analysis of the data; AG, PC, SM: drafted the article; FP: critically revised the article and gave final approval of the version to be published. All Authors critically revised the article, approved the final version to be published, and agree to be accountable for all aspects of the work. 


\section{References}

1 WHO: Novel Coronavirus (2019-nCoV). Situation report-1 21, Jan 2020. Available at: https://www.who.int/docs/default-source/ coronaviruse/situation-reports/20200121-sitrep-1-2019-ncov.pdf

2 Tuscany Regional Agency of Health guidelines: ARS TOSCANA-agenzia regionale di sanità; 29, Jun 2020. Available at: https://www.ars.toscana.it

3 Baumann M, Krause M, Overgaard J, Debus J, Bentzen SM, Daartz J, Richter C, Zips D and Bortfeld T: Radiation oncology in the era of precision medicine. Nat Rev Cancer 16(4): 234-249, 2016. PMID: 27009394. DOI: $10.1038 /$ nrc.2016.18

4 Fowler JF and Lindstrom MJ: Loss of local control with prolongation in radiotherapy. Int J Radiat Oncol Biol Phys 23(2): 457-467, 1992. PMID: 1534082. DOI: 10.1016/0360-3016(92) 90768-d
5 Italian Association of Radiotherapy and Oncology: Guidlines for the risk assessment and management of patients and operators in the oncological radiotherapy wards in the process of spreading Covid-19. Available at: https://www.radioterapiaitalia.it/wpcontent/uploads/2020/03/v-2-Documento-AIRO-COVID-19-2403-2020.pdf

6 Guidelines from the President of the Tuscany Regional Council n. 23. Available at: http://www301.regione.toscana.it/bancadati/ atti/Contenuto.xml?id=5251885\&nomeFile=Ordinanza_del_Presid ente_n.23_del_03-04-2020

Received September 1, 2020

Revised October 5, 2020

Accepted October 8, 2020 